\title{
Reverse-emission-state-transition mode locking of a two-section InAs/ InGaAs quantum dot laser
}

\author{
Stefan Breuer, ${ }^{1, a)}$ Mattia Rossetti, ${ }^{2}$ Wolfgang Elsässer, ${ }^{1}$ Lukas Drzewietzki, ${ }^{1}$ \\ Paolo Bardella, ${ }^{2}$ Ivo Montrosset, ${ }^{2}$ Michel Krakowski, ${ }^{3}$ and Mark Hopkinson ${ }^{4}$ \\ ${ }^{1}$ Institute of Applied Physics, Technische Universität Darmstadt, 64289 Darmstadt, Germany \\ ${ }^{2}$ Dipartimento di Elettronica, Politecnico di Torino, 10129 Torino, Italy \\ ${ }^{3}$ Thales-Alcatel III-V Labs, 91767 Palaiseau, France \\ ${ }^{4}$ Department of Electronic and Electrical Engineering, University of Sheffield, Sheffield S1 3JD, \\ United Kingdom
}

(Received 25 June 2010; accepted 28 July 2010; published online 19 August 2010)

\begin{abstract}
Reverse-emission-state-transition mode locking in a two-section InAs/InGaAs quantum dot laser is experimentally investigated and confirmed by simulations. Stable mode locking starts on the first excited state $(\lambda=1207 \mathrm{~nm})$ and then, with increasing gain current, a transition to stable simultaneous two-state mode locking on excited state and ground state $(\lambda=1270 \mathrm{~nm})$ takes place. This particular state-transition occurs already at $0 \mathrm{~V}$ reverse-bias and at moderate gain-section currents. It is attributed to the strong active region chirping of the gain medium and in particular to a photon pumping process in the saturable absorber section. (c) 2010 American Institute of Physics. [doi:10.1063/1.3480405]
\end{abstract}

Quantum dot (QD) semiconductor lasers exhibit, besides their immediate application potential, ${ }^{1}$ particularly interesting dynamical behavior due to the existence of ground state (GS) and excited state (ES) in the quantized dot energy scheme originating from the interaction between these two states and the quasi-two-dimensional wetting layer. ${ }^{2-6}$ One particularity which shows this complex interplay is the recent observation of dual-wavelength mode locking (ML) in an AR/HR coated InAs/GaAs two-section QD laser. ${ }^{7}$ A transition from GS to ES ML was reported with increasing gain current together with a coexistence regime of GS ML and ES ML at gain currents larger than $300 \mathrm{~mA}$ and at reverse-bias voltages between 6 to $10 \mathrm{~V}$. In an appropriately designed uncoated two-section QD structure a resistor self-electrooptical effect (SEED) has recently been demonstrated where among others a reversal of the emission-state-transition was observed. ${ }^{8}$ ML started on the ES and continuous-wave emission from the GS joined the ES ML with increasing gain current.

Here, we present detailed joint experimental and theoretical investigations of reverse-emission-state-transition dynamics covering mode-locked operation of both involved states at $0 \mathrm{~V}$ reverse-bias and identify the basic physical origins of this ML regime.

The QD active region consists of a multilayer dot-in-awell (DWELL) structure in which InAs dots, grown by Stranski-Krastanov process via MBE, are embedded in an InGaAs quantum well (QW). To obtain a broad gain spectrum the QD multilayer has been chirped. The active layer contains two repeats of three chirped layers and is embedded in GaAs confinement layers and GaAlAs waveguide cladding layers. By modifying the Indium content of the InAs QD surroundings within the QW the inhomogeneous broadening of the DWELL structure is enhanced beyond that of a single QD ensemble and the overall device exhibits a gain bandwidth in the region of $(80-100) \mathrm{nm}^{9}$ A narrow ridge

${ }^{a)}$ Electronic mail: stefan.breuer@physik.tu-darmstadt.de. two-section laser with a $3 \mathrm{~mm}$ long gain section and a 0.3 $\mathrm{mm}$ long saturable absorber (SA) section was fabricated. The facets were as cleaved. The gain section is pumped with a current source and the absorber section is connected to a voltage source. The laser is operated at $20^{\circ} \mathrm{C}$. Spectral characterization is performed by an optical spectrum analyzer and $\mathrm{ML}$ is investigated by a $50 \mathrm{GHz}$ photodiode in combination with an electrical spectrum analyzer and by an highsensitivity second-harmonic-generation auto-correlator.

According to the biasing conditions of the gain and the SA section a lasing state hierarchy exists. For electrically connecting gain and SA section together the threshold current amounts to $40 \mathrm{~mA}$ and lasing occurs on the GS only at $1270 \mathrm{~nm}$. Under open circuit condition of the SA, the threshold current amounts to $90 \mathrm{~mA}$ and laser emission on the GS is observed solely. However, by applying $0 \mathrm{~V}$ to the SA, a reversal of the emission-state-transition occurs: stable lasing and ML starts at the ES transition.

The experimentally measured spectral evolution of this reverse-emission-state-transition ML is depicted in Fig. 1.

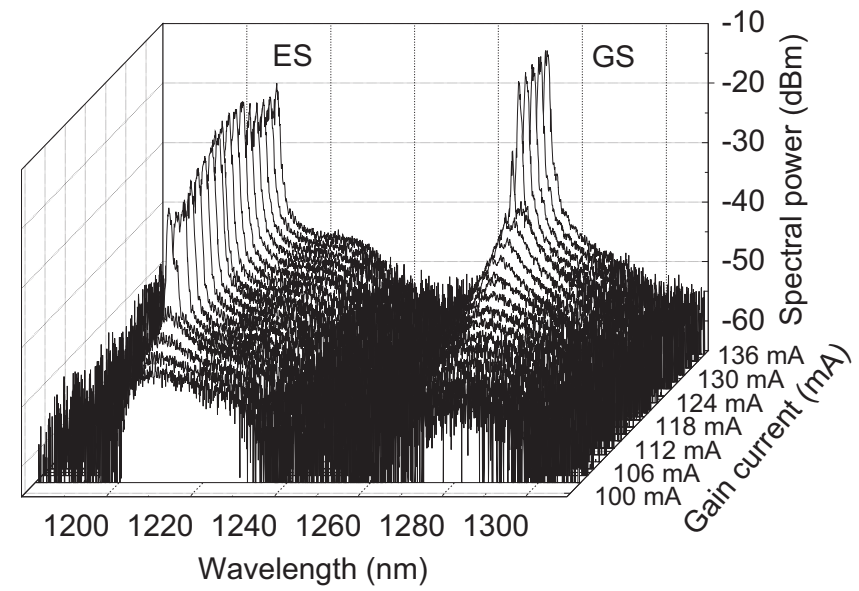

FIG. 1. Three-dimensional depiction of the experimentally obtained optical spectra evolution from $100 \mathrm{~mA}$ to $136 \mathrm{~mA}$ and for $0 \mathrm{~V} \mathrm{SA}$ bias. 


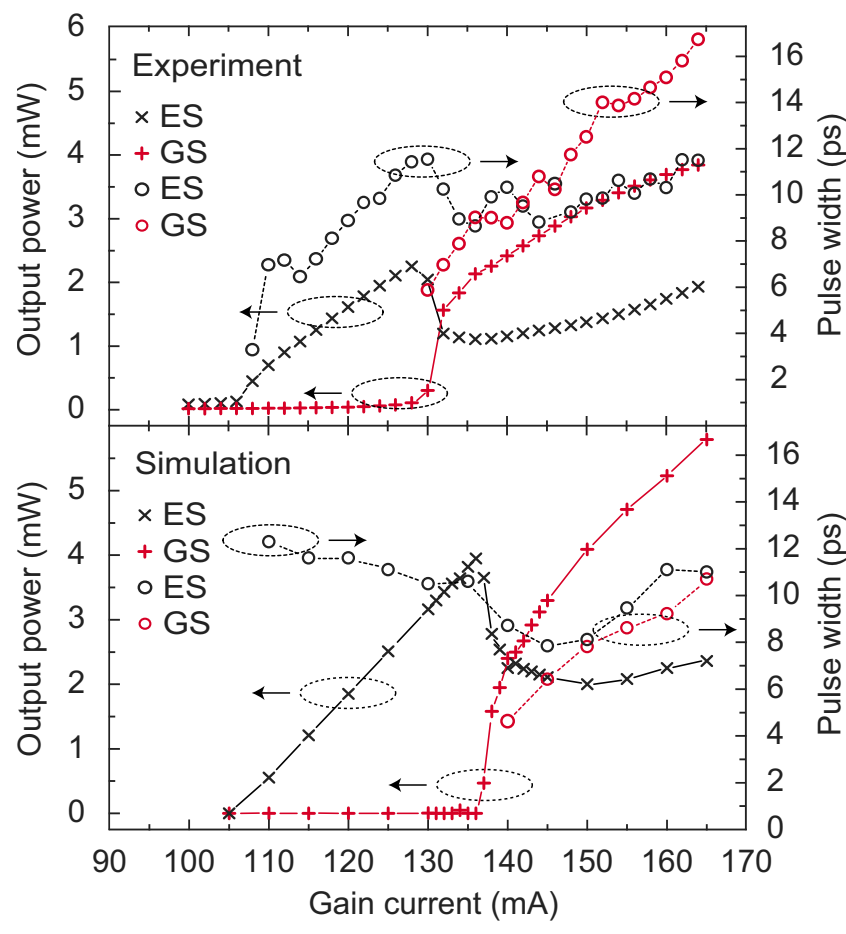

FIG. 2. (Color online) Experimentally obtained and simulated state-resolved output-power characteristics (left scale) and pulse widths (right scale) vs gain section current for the ES and the GS transition for 0 V SA bias. Top: experimental results. Bottom: simulation results.

The ES ML threshold occurs at $105 \mathrm{~mA}$ and stable ES ML is obtained up to $130 \mathrm{~mA}$. Above $130 \mathrm{~mA}$, additional to the ES ML, lasing and ML on the GS occurs. Both states coexist leading to a stable two-state ML regime. The corresponding wavelength- or state-resolved total laser output-power characteristics together with the pulse widths are shown in Fig. 2 (top). These results have been obtained for $0 \mathrm{~V}$ SA bias and by inserting interference filters in the experimental set-up. Starting at $110 \mathrm{~mA}$, a continuous increase in the ES pulse width from 3.3 to 11.5 ps occurs. With the onset of GS ML at $130 \mathrm{~mA}$, the ES pulse width decreases appreciably to $8.6 \mathrm{ps}$ with a subsequent re-increase to $11.5 \mathrm{ps}$ at $165 \mathrm{~mA}$. The GS ML starts with a shorter pulse width of 6 ps as compared to the ES pulse width at the same gain current, then crosses the ES pulse width and increases to $16.7 \mathrm{ps}$ at $165 \mathrm{~mA}$. Above the corresponding state threshold, stable ML of the respective state occurs. The simultaneity of this two-state ML operation is experimentally confirmed via the occurrence of intermodulation (IM) signals of the beats of ES and GS in the radio-frequency $(\mathrm{RF})$ beat spectra shown in Fig. 3 for two different gain currents. ${ }^{10}$ At $128 \mathrm{~mA}$, below the GS ML threshold where only the ES ML takes place, one single beat at the cavity round trip frequency of $12.096 \mathrm{GHz}$ is observed. For a gain current of $132 \mathrm{~mA}$, above the GS ML threshold, the ES beat and the GS beat now occur at frequencies of $12.105 \mathrm{GHz}$ and $12.321 \mathrm{GHz}$, respectively. The longer wavelength of the GS results in a higher beat frequency due to dispersion. Also clearly visible are IM signals of GS and ES beats resulting in frequency sidebands at 11.89 $\mathrm{GHz}$ and $12.538 \mathrm{GHz}$, respectively. These beats are also transferred to the low-frequency band at $237 \mathrm{MHz}$ as shown in the inset of Fig. 3. This is a clear evidence of the simultaneity of GS and ES mode-locked operation.

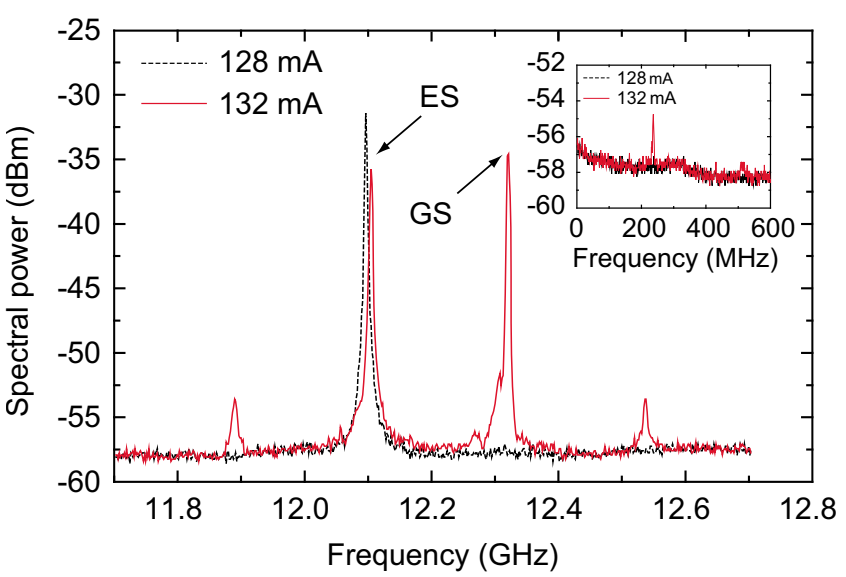

FIG. 3. (Color online) Experimentally obtained RF beat spectra of the mode-locked pulses for a gain current of $128 \mathrm{~mA}$ (dashed), below the GS ML threshold, and $132 \mathrm{~mA}$ (solid), above the GS ML threshold, and for $0 \mathrm{~V}$ SA bias. The inset shows the low-frequency beat domain.

The particular reverse-emission-state-transition dynamics is attributed to the designed properties of the realized laser structure in conjunction with the employed SA biasing conditions, i.e., a small number of QD layers and, in particular, a gain bandwidth that is strongly broadened by activeregion chirping such that the maximum saturated net modal gain at the GS peak wavelength is significantly reduced. A comparable device with a narrower chirping does not show this particular behavior.

To simulate this transition regime in two-section QD lasers, a finite-difference time-domain traveling-wave model is implemented describing the evolution of the forward and backward traveling optical power along the laser cavity at both GS and ES wavelengths. ${ }^{11-13}$ Carrier dynamics in the QD layers are described via a dedicated set of rate-equations in the excitonic approach allowing to properly reproduce gain and absorption dynamics in the gain and the SA section, respectively. It was found that gain and absorption dynamics in the gain and the SA section are the main factor leading to the experimentally observed ML regime. In fact, incorporating finite gain spectral bandwidth, self-phase modulation and dispersion in the simulations only led to minor differences in the ML regime. ${ }^{11,14}$ Therefore, and to improve computational time, these effects have been omitted in the simulations. Intrinsic waveguide losses of $3.5 \mathrm{~cm}^{-1}$ and a low maximum saturated net modal gain of $8 \mathrm{~cm}^{-1}$ for the GS and $13 \mathrm{~cm}^{-1}$ for the ES are required in the simulations in order to obtain reverse-emission-state-transition leading to stable simultaneous two-state ML at $0 \mathrm{~V} \mathrm{SA}$ bias and at moderate gain currents. The simulated state-resolved power-current characteristics at $0 \mathrm{~V} \mathrm{SA}$ bias are shown in Fig. 2 (bottom). Consistent with the experiments, the onset of GS lasing is achieved after the ES threshold. By further increasing the current both GS and ES lasing coexist and a stable dual-state ML is obtained. In the simulations, just after the ES threshold, the ES pulse width slightly decreases with current. This behavior contrasts with the increase in the ES pulse width found in the experiments. The discrepancy may be due to a difference between the actual ES absorption saturation energy and the effective one obtained from the simulations that depends on the complex carrier dynamics between the QD confined states in the SA section; further investigations of this behavior are ongoing. At the onset of two-state ML, a 
good qualitative agreement between the simulated and experimental behavior of both GS and ES pulse width and optical power is instead obtained: appreciably shorter pulse widths are found for the GS as compared to the ES at the same gain current and a considerable shortening of the ES pulse widths is observed when GS ML starts; finally increasing further the applied current, both GS and ES pulses tend to broaden.

Despite the lack of a complete quantitative agreement, the simulations still give insight into the onset of the experimentally observed ML regime. In particular, it becomes evident that the onset of additional GS ML is mainly due to a so-called photon pumping or photon recycling process in the SA section. The photons generated at the ES wavelength are absorbed in the SA section. The fast relaxation of ES carriers in the GS then leads to a reduction in the absorption at the GS wavelength in the SA. If this effect is strong enough, the onset of GS emission and even GS ML is obtained, guaranteeing the coexistence of GS and ES ML over a wide range of gain currents. Such an interplay has been proposed in Ref. 15 in the case of a two-section QD laser operating in continuous-wave or self-pulsating regime. The simulations identify a sufficiently low GS gain and the photon pumping in the SA section as the main processes to obtain reverse-emission-state-transition ML together with stable simultaneous two-state ML.

In this contribution a two-section InAs/InGaAs QD laser structure has been experimentally investigated and simulated that exhibits reverse-emission-state-transition ML. Stable ML starts first on the energetically higher-lying ES and then, with increasing gain current, a transition to stable simultaneous two-state ML on both ES and GS takes place. The simultaneity of this two-state ML operation has been experimentally confirmed. The observed reverse-emission-state dynamics has been substantially verified by time-domain traveling-wave equation modeling and attributed to the significantly broadened gain spectrum caused by strongly chirped QD layers which results in a small saturated net modal gain and, in particular, to a photon pumping process in the SA section. This particular QD structure allows tailored two-state ML, potentially interesting for applications requir- ing picosecond pulse operation at two different wavelengths for telecommunication, spectroscopy and biomedical imaging.

Funding by the EU Sixth Framework Program project 'NANO UB-SOURCES' (Grant No. 017128) and the EU Seventh Framework Program 'FAST-DOT' (Grant No. 224338) is gratefully acknowledged.

${ }^{1}$ D. Bimberg, in Optical Fiber Telecommunications V A: Components and Subsystems, edited by I. Kaminov, T. Li, and A. Willner, 2008), Chap. 2, p. 23.

${ }^{2}$ E. U. Rafailov, M. A. Cataluna, and W. Sibbett, Nat. Photonics 1, 395 (2007).

${ }^{3}$ A. Markus, J. X. Chen, C. Parantheon, A. Fiore, C. Platz, and O. GautierLafaye, Appl. Phys. Lett. 82, 1818 (2003).

${ }^{4}$ E. A. Viktorov, M. A. Cataluna, L. O’Faolain, T. F. Krauss, W. Sibbett, E. U. Rafailov, and P. Mandel, Appl. Phys. Lett. 90, 121113 (2007).

${ }^{5}$ M. A. Cataluna, W. Sibbett, D. A. Livshits, J. Weimert, A. R. Kovsh, and E. U. Rafailov, Appl. Phys. Lett. 89, 081124 (2006).

${ }^{6}$ D. I. Nikitichev, M. A. Cataluna, I. Krestnikov, D. Livshits, and E. U. Rafailov, Proceedings of the first EOS Topical Meeting on Lasers, 2243 Capri, Italy, 2009.

${ }^{7}$ M. A. Cataluna, D. I. Nikitchev, S. Mikroulis, H. Simos, C. Mesaritakis, C. Syvridis, I. Krestnikov, D. Livshits, and E. U. Rafailov, Opt. Express 18, 12832 (2010).

${ }^{8}$ S. Breuer, W. Elsässer, and M. Hopkinson, Electron. Lett. 46, 161 (2010).

${ }^{9}$ S. K. Ray, K. M. Groom, M. D. Beattie, H. Y. Liu, M. Hopkinson, and R. A. Hogg, IEEE Photonics Technol. Lett. 18, 58 (2006).

${ }^{10}$ Simultaneity means that the laser is not switching between the two states. In a different laser structure very recently also an interesting bi-stable switching at the GS transition has been reported: F. Mingming, S. T. Cundiff, R. P. Mirin, and K. L. Silverman, IEEE J. Quantum Electron. 46, 951 (2010).

${ }^{11}$ P. A. Morton, R. J. Helkey, and J. E. Bowers, IEEE J. Quantum Electron. 25, 2621 (1989).

${ }^{12}$ S. Breuer, M. Rossetti, W. Elsässer, L. Drzewietzki, P. Bardella, I. Montrosset, M. Krakowski, and M. Hopkinson, Proc. SPIE 7720, 772011 (2010).

${ }^{13}$ M. Rossetti, P. Bardella, I. Montrosset, S. Breuer, and W. Elsässer, Proceedings of the 15th European Conference on Integrated Optics, ThP09, 2010.

${ }^{14}$ M. Rossetti, P. Bardella, and I. Montrosset, IEEE J. Quantum Electron. "Time-domain traveling-wave model for quantum dot passively modelocked lasers" (to be published).

${ }^{15}$ A. Markus, M. Rossetti, V. Calligari, D. Chek-Al-Kar, J. X. Chen, and A. Fiore, J. Appl. Phys. 100, 113104 (2006). 
Applied Physics Letters is copyrighted by the American Institute of Physics (AIP). Redistribution of journal material is subject to the AIP online journal license and/or AIP copyright. For more information, see http://ojps.aip.org/aplo/aplcr.jsp 